\section{§24. Transition in Multiple-scale-lengths} Turbulence in Plasmas

Itoh, S.-I. (RIAM, Kyushu Univ.), Itoh, K.

Statistical theory of strong turbulence in inhomogeneous plasmas is extended to analyzing the state where fluctuations with different scale lengths coexist. The nonlinear interactions between micro-mode (being represented by, e.g., current-diffusive interchange mode (CDIM) of the scale length $\sim \delta$, the skin depth, or by the electron temperature gradient (ETG) mode) and semimicro mode (e.g., drift mode or ion temperaturegradient (ITG) mode of $\sim \rho_{i}$, the ion gyroradius) are taken into account for the case of $\delta<\rho_{i}$. The nonlinear interactions of two collective modes of fluctuations are treated as follows. The microturbulence of smaller size affects the semi-micro one through the renormalized drag and the "micro"-noise. The effect of semi-micro" turbulence is included in the dynamical equation of the micro-mode as a kind of a driving force or damping force. Nonlinear self-interactions in one class of fluctuation are divided into the coherent drag and the self-noise. The combined Langevin equations are to be formulated. A set of equations of the fluctuation level and decorrelation rate for the micro and semi-micro fluctuations is obtained. A case where two driving mechanisms exist (one for micro mode and the other for semi-micro mode) is investigated.

We consider the situation where two kinds of fluctuations are simultaneously excited. Scale separation is introduced. Their scale lengths are assumed to be different, and the semi-micro component and micro one are denoted by symbols $\mathrm{l}$ (low) and h (high), respectively. According to the methodology of [1], a set of nonlinear equations for the spectral intensities $I^{h, l}=\sum_{k}\left\langle\phi_{1, k}^{h, l} \phi_{1, k}^{h, l}\right\rangle$ and the eddy damping rates $\gamma_{v}^{h, l}$ are obtained.as

$$
\begin{aligned}
& \frac{\gamma_{v}^{l^{2}}}{k_{0}^{l 4}} \simeq I^{l}+\frac{\gamma_{v}^{l}}{k_{0}^{l 2}} \sqrt{I^{h}} . \\
& \frac{\gamma_{v}^{l}}{k_{0}^{l 2}} I^{l}=D^{l} I^{l}+\varepsilon\left(I^{h}\right)^{3 / 2}
\end{aligned}
$$

where $D^{l}=2\left(2-C_{0}^{l}\right)^{-1}\left(1+\left(\omega_{E} / \omega_{E c}^{l}\right)^{2}\right)^{-1} \gamma_{0}^{l} k_{0}^{l-2}$ and $\varepsilon=\hat{C}_{0}^{h}\left(2-C_{0}^{l}\right)^{-1}\left(k_{0}^{l} / k_{0}^{h}\right)^{2}$ for semi-micro mode, $\left(\omega_{E}\right.$ denote the radial electric field inhomogeneity) and

$$
\begin{aligned}
& \gamma_{v}^{h} \simeq k_{0}^{h^{2}} \sqrt{I_{i}^{h}} \\
& I^{h}=\left(D^{h}\right)^{2} \frac{1+\left|k_{0}^{l 2} / 2 \gamma_{v}^{l}\right| \sqrt{I^{l}}}{\left(1+I_{\text {eff }}^{-1} I^{l}\right)^{2}}
\end{aligned}
$$

where $D^{h}=2\left(2-C_{0}^{h}\right)^{-1}\left(1+\left(\omega_{E} / \omega_{E c}^{h}\right)^{2}\right)^{-1} \gamma_{0}^{h} k_{0}^{h-2}$, and $I_{\text {eff }} \equiv\left(1+\left(\omega_{E} / \omega_{E C}^{h}\right)^{2}\right)\left(\omega_{E C}^{h}\right)^{2}\left(k^{l}\right)^{-4}$ for micro mode. Here, $D^{l}$ and $D^{h}$ denote the magnitude of the driving power by the global inhomogeneity and represent the characteristic level of diffusivity of semi-micro mode and that for micro mode, respectively. In the absence of the mutual nonlinear interactions, we have $I^{l} \simeq\left(D^{l}\right)^{2}$ and $I^{h} \simeq\left(D^{h}\right)^{2} . I_{\text {eff }}$ represents the level of semi-micro mode which is enough to suppress the micro mode by velocity shear.

Equations (1) and (2) have multiple solutions and show the hard bifurcation [2]. Figure 1 illustrates the fluctuation amplitude as a function of $D^{l}$ for the fixed value of $D^{h}$. $\left(\sqrt{I_{\text {eff }}}=0.5 D^{h}\right)$ When the drive for the semi-micro mode is weak, $D^{l}<D^{h}$, the micro mode is excited, but the semi-micro mode is quenched. For $D^{l}>D^{h}$, the micro mode is suppressed.

In summary, the statistical theory of strong turbulence in inhomogeneous plasmas was extended to analyzing the nonlinear interactions when fluctuations with different scale lengths coexist. A new turbulence transition was obtained, being associated with a hard bifurcation.

\section{References}

1) Itoh S-I and Itoh K: J. Phys. Soc. Jpn. 68 (1999) 1891; 2611; 69 (2000) 408; 427; 3253. 2) Itoh S-I and Itoh K Plasma Phys. Contr. Fusion $43(2001)$

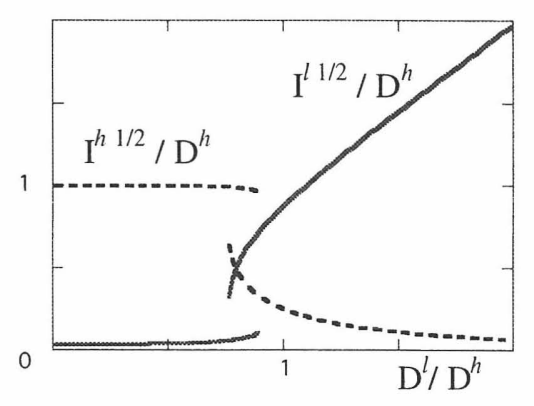

Fig.1 Fluctuation level for semi-micro mode (solid line) and that for micro mode (dashed line) as a function of the driving rate of the semi-micro mode. Driving rate for the micro mode is fixed. 